\title{
THERE ARE ASYMPTOTICALLY FAR FEWER POLYTOPES THAN WE THOUGHT
}

\author{
BY JACOB E. GOODMAN ${ }^{1}$ AND RICHARD POLLACK ${ }^{2}$
}

The problem of enumerating convex polytopes with $n$ vertices in $\mathbf{R}^{d}$ has been the object of considerable study going back to ancient times (see $[4, \S 13.6]$ for some remarks about the history of this problem since the nineteenth century). While significant progress has been made when the number of vertices was not too much larger than the dimension [4], little had been known above dimension 3 in the general case despite considerable efforts: if $f(n, d)$ is the logarithm of the number of combinatorially distinct simplicial polytopes with $n$ vertices in $\mathbf{R}^{d}$, the sharpest asymptotic bounds previously known for $f(n, d)$ were

$$
c_{1} n \log n<f(n, d)<c_{2} n^{d / 2} \log n,
$$

with the lower bound due to Shemer [7], and the upper bound following from the (asymptotic) Upper Bound Theorem of Klee [5], leaving a wide gap between the two bounds. (Here, and in the sequel, we take the view that $d$ is fixed and $n \rightarrow \infty$; thus all constants depend on $d$.) The purpose of the present note is to announce a considerable narrowing of this gap:

THEOREM. $c_{1} n \log n<f(n, d)<c_{3} n \log n$.

The proof of the new upper bound is based on results of Milnor [6] and Goodman-Pollack [2]. An outline follows; details, as well as a related result on the number of combinatorial equivalence classes of labelled point configurations, will appear in [3].

Step 0. Instead of considering isomorphism classes of simplicial polytopes, we consider isomorphism classes of labelled simplicial polytopes, i.e., polytopes whose vertices are numbered, modulo only those isomorphisms of the simplicial structure which respect the numbering. This introduces a factor of $n$ ! (or less, depending on the order of the symmetry group), hence-by Stirling's formula-does not affect the result.

Step 1. We suppress the simplicial structure of each polytope under consideration, and consider only the order type of its set of vertices $P_{1}, \ldots, P_{n}$; the order type of a configuration of $n$ points in $\mathbf{R}^{d}$ is its equivalence class under the relation

$$
\left\{P_{1}, \ldots, P_{n}\right\} \sim\left\{Q_{1}, \ldots, Q_{n}\right\}
$$

Received by the editors August 25, 1985.

1980 Mathematics Subject Classification. Primary 52A25; Secondary 05A15, 14G30, $51 \mathrm{M} 20$.

${ }^{1}$ Supported in part by NSF Grant DMS-8501492 and PSC-CUNY Grant 665258.

${ }^{2}$ Supported in part by NSF Grant DMS-8501947. 
if and only if

$$
\left\{P_{i_{0}}, \ldots, P_{i_{d}}\right\} \text { and }\left\{Q_{i_{0}}, \ldots, Q_{i_{d}}\right\}
$$

have the same orientation for every $d+1$-tuple $i_{0}, \ldots, i_{d}$. The order type of a configuration determines the sets of vertices which form the facets of its convex hull [2], hence-in this case-the entire simplicial structure of the original polytope. Moreover, by perturbing the vertices slightly, we can assume that they lie in general position. Thus the problem of finding an upper bound for $f(n, d)$ reduces to the problem of bounding the function $g(n, d)$, the logarithm of the number of distinct order types of simple labelled configurations of $n$ points in $\mathbf{R}^{d}$.

Step 2. To each labelled configuration $S=\left\{\left(x^{1}\right), \ldots,\left(x^{n}\right)\right\}$ of points in $\mathbf{R}^{d}$ we associate a point $(x) \in \mathbf{R}^{d n}$. The order type of $S$ can then be viewed as a mapping

$$
\omega: \quad \mathbf{R}^{d n} \rightarrow\{-1,0,1\}^{\left(\begin{array}{c}
n \\
d+1
\end{array}\right)}
$$

with $\omega$ defined by

$$
\omega\left(\left(x^{1}\right), \ldots,\left(x^{n}\right)\right)=\left(\operatorname{sgn} \operatorname{det}\left(\begin{array}{cccc}
1 & x_{1}^{i(0)} & \cdots & x_{d}^{i(0)} \\
& & \cdots & \\
1 & x_{1}^{i(d)} & \cdots & x_{d}^{i(d)}
\end{array}\right)\right)_{1 \leq i(0)<\cdots<i(d) \leq n}
$$

(see [2] for details). To say that $S$ is simple means that

$$
\omega(S) \in\{-1,1\}^{\left(\begin{array}{c}
n \\
d+1
\end{array}\right)},
$$

i.e., none of the determinants above vanishes at the point corresponding to $S$.

Each of these determinants is a polynomial of degree $d$ in the $d n$ variables $X_{1}^{1}, \ldots, X_{d}^{n}$, so if we multiply them we get a single polynomial

$$
P\left(X_{1}^{1}, \ldots, X_{d}^{n}\right) \text { of degree } d\left(\begin{array}{c}
n \\
d+1
\end{array}\right),
$$

whose zero locus $V$ corresponds precisely to the set of nonsimple configurations. Let $U$ be the complement of $V$. Then a connected component of $U$ is a full isotopy class of simple configurations, i.e., a maximal set such that any two can be deformed, one into the other, by a continuous family of configurations all having the same order type. In particular, $g(n, d)$ is bounded above by the logarithm of the number of connected components of $U$.

Further reductions, in which the strong inequalities which define $U$ are replaced by suitable weak inequalities, bring about a situation in which we can apply the following theorem of Milnor on the cohomology of semialgebraic sets.

THEOREM [6]. If a set $X \subset \mathbf{R}^{m}$ is defined by polynomial inequalities of the form

$$
f_{1} \geq 0, \quad \ldots, \quad f_{p} \geq 0
$$

of total degree $d=\operatorname{deg}\left(f_{1}\right)+\cdots+\operatorname{deg}\left(f_{p}\right)$, then

$$
\operatorname{rank} H^{*} X \leq(1 / 2)(2+d)(1+d)^{m-1} \text {. }
$$


In particular, this implies that the number of connected components of our set $U$ is bounded above by

$$
\left(2+d\left(\begin{array}{c}
n \\
d+1
\end{array}\right)\right)\left(1+d\left(\begin{array}{c}
n \\
d+1
\end{array}\right)\right)^{d n-1},
$$

from which the main result follows.

The argument above shows that the constant $c_{3}$ can be taken to be $d^{2}(1+$ $1 / d)$. This has recently been improved to $d^{2}(1+o(1))$ by $\mathrm{N}$. Alon [1], using a clever modification of the argument outlined here, who shows moreover that the bound continues to hold even if the assumption that the polytopes are simplicial is removed.

\section{REFERENCES}

1. N. Alon, The number of polytopes, configurations, and real matroids, preprint.

2. J. E. Goodman and R. Pollack, Multidimensional sorting, SIAM J. Comput. 12 (1983), 484-507.

3. J. E. Goodman and R. Pollack, Upper bounds for configurations and polytopes in $\mathbf{R}^{d}$, Discrete Comp. Geom. (to appear).

4. B. Grünbaum, Convex polytopes, Interscience-Wiley, London, 1967.

5. V. Klee, The number of vertices of a convex polytope, Canad. J. Math. 16 (1964), 701-720.

6. J. Milnor, On the Betti numbers of real varieties, Proc. Amer. Math. Soc. 15 (1964), 275-280.

7. I. Shemer, Neighborly polytopes, Israel J. Math. 43 (1982), 291-314.

Department of Mathematics, City College (CUNy), New York, New YORK 10031

Department of MAThematics, Courant institute (NYU), NeW York, NEW YORK 10012 
